\title{
The use of botulinum toxin in the management of bruxism and facial arthromyalgia syndrome
}

\author{
Raluca A. DRAGOMIR ${ }^{1}$, Cristian Ilie DROCHIOI ${ }^{1}$, Alexandra CARP ${ }^{1}$, \\ Bogdan DRAGOMIR ${ }^{2}$, Otilia BOISTEANU ${ }^{1}$, Victor-Vlad COSTAN ${ }^{1}$ \\ ${ }^{1}$ Oral and Maxillofacial Surgery Department, \\ "Grigore T. Popa" University of Medicine and Pharmacy, lasi, Romania \\ ${ }^{2}$ Orthodontics and Facial Orthopedics, \\ "Grigore T. Popa" University of Medicine and Pharmacy, Iasi, Romania
}

\begin{abstract}
Objectives. The focus of this study is to determine the outcomes of botulinum toxin injections in patients with symptomatic bruxism. Material and methods. We reviewed the medical records of patients with bruxism and facial arthromyalgia syndrome treated by botulinum toxin injections. We evaluated the initial complaints, clinical findings, technique of neurotoxin administration, previous treatments and post-injection outcomes. Results. Pain related symptoms were significantly decreased in all patients one month following administration and maintained three months post-injection. Complications of the technique were mild and rarely encountered. Conclusions. Botulinum toxin is effective in reducing the associated symptoms of bruxism, and its administration is generally safe if careful injection technique is employed and adequate doses used.
\end{abstract}

Keywords: bruxism, botulinum toxin, arthromyalgia, temporomandibular joint, injection, masseter

\section{INTRODUCTION}

The spectrum of indication for botulinum toxin administration gradually increased over time as new benefits of this treatment were discovered, with wide applications ranging from cosmetic purposes, to treating muscular contractures, spasticity, dystonia and even neuromuscular pain (1-4).

The effects of botulinum toxin administration were attributed mainly to the well-established property of inhibiting the release of acetylcholine at the synapse level, therefore inducing absence of stimulation of the effector, whether it is a muscle or a gland with resulting obvious benefits in the treatment of conditions involving such structures (1). Additionally, multiple studies underlined the effects of botulinum toxin concerning the inhibition of the release of several mediators of inflammation and peripheral neurotransmitters from the sensory nerve fibers, resulting in decreased pain (5-7).

Considering multiple interrelating mechanisms involved in the development of bruxism and facial arthromyalgia syndrome, including neuro-muscular, structural, mechanical, behavioral and psy- 
chological factors, botulinum toxin may prove a beneficial contributor in decreasing the symptomatology and increasing the quality of life of affected patients $(8,9)$. The input of the neurotoxin administration in the multifactorial context of the disease has been the focus of many studies, but further research is still required to establish the outcomes of its clinical use in different scenarios $(10,11)$.

The purpose of this study is to evaluate the effects of botulinum toxin administration in patients with bruxism and facial arthromyalgia syndrome, with previous conventional treatment attempts, regarding the improvement of clinical signs and symptoms.

\section{MATERIAL AND METHODS}

We conducted a retrospective study including patients with known bruxism and facial arthromyalgia syndrome that underwent treatment by botulinum toxin injection. We included only adult patients with minimum 3 months of follow-up. Patients with declared personal history of trauma or surgery of the temporomandibular joint (TMJ) were excluded from the study. The presenting signs and symptoms were documented, as well as the injection technique, associated treatment measures and the reported outcomes at two weeks, one and three months following botulinum toxin administration.

\section{RESULTS}

\section{Patients}

We included 17 patients in this study, eight men and nine women, aged between 19 and 39 years old. The patients declared symptoms like local TMJ pain ( $\mathrm{n}=15)$, masticatory muscle pain $(n=14)$, referred otalgia $(n=6)$, cephalalgia $(n=$ $9)$. Most patients $(n=12)$ declared the predominance of symptoms in the morning, while five noticed the occurrence of symptoms during the day. Duration of the reported symptoms before presentation was between 11 and 32 months. Eleven patients corelated the onset of symptoms with stressful events. From the total number of patients, six were previously diagnosed with anxiety disorders and five had depression. Clinical examination revealed the presence of temporomandibular joint pain upon palpation in nine cases, joint sounds in seven patients, limited jaw opening in six cases, painful muscle insertions in 11 patients, muscle hypertrophy in nine cases, tooth surface loss in 12 patients and important occlusal disturbances in five cases.

All patients had previous treatment attempts by one or several of the following methods: occlusal therapy $(n=7)$, occlusal splints $(n=14)$, administration of myorelaxants $(n=17)$, antidepressants ( $n=9)$, antalgics ( $n=13$ ) and behavioral changes recommendations ( $n=17)$. The conservative treatment methods were continued after the neurotoxin administration.

\section{Injection technique}

Neurotoxin injections were performed in the masseter and temporalis muscles, bilaterally, in all patients, using Vistabelâ (Onabotulinumtoxin type A, Allergan Pharmaceuticals Ireland, Castlebar Road, Westport, County Mayo, Ireland). We used $1.25 \mathrm{ml}$ of saline for a vial of $50 \mathrm{U}$, to achieve a dilution of $4 \mathrm{U}$ per $0.1 \mathrm{ml}$. The total doses varied between 100 and $200 \mathrm{U}$ for one patient, distributed into multiple injection sites, with a minimum of five injection points for the masseter muscle and three for the temporalis muscle on one side. We used syringes of $1 \mathrm{ml}$ with a detachable $27 \mathrm{G}$ $19-\mathrm{mm}$ needle. The areas of muscle hyperactivity were initially palpated and marked. For administering the injection, the needle was inserted up to the level of the bone and then withdrawn for 2-4 $\mathrm{mm}$, followed by the deposition of the botulinum toxin solution.

\section{Injection outcomes}

The maximum results were reported two weeks post-injection. Eleven patients reported an overall improvement of pain related symptoms that was maintained one month and three months post-injection. The other six patients reported no initial changes regarding pain perception first couple of weeks but noted a decrease in pain one month and three months post-injection. Clinical examination one month and three months revealed the decreased number of painful sites upon palpation of masticatory muscles in 12 patients and the amplitude of mouth opening was normal in all patients. TMJ sounds were still present in six patients at three months post-injection, but absence of pain. At three months, we noticed a decrease in the contour of the masseteric region in 14 cases (figures 1, 2).

In three patients minor bruising in the masseteric area occurred after the injection. Following the administration of neurotoxin all patients reported feeling masticatory muscle fatigue during the first month, with progressive function adaptation. Two patients declared a subjective dry mouth 


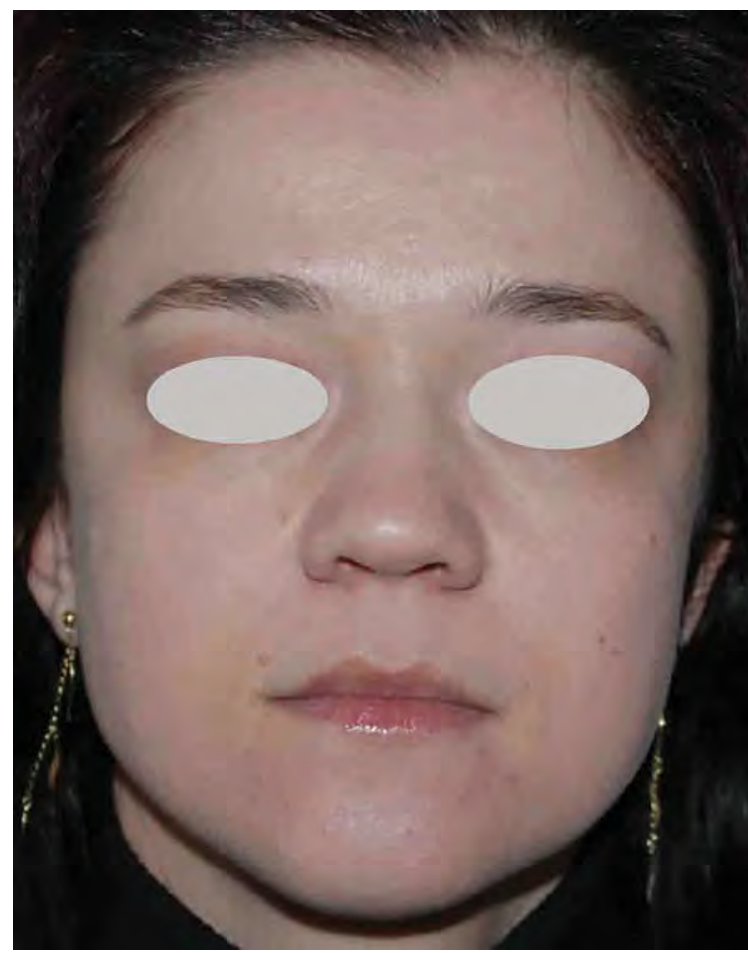

FIGURE 1. Patient with bruxism, facial arthromyalgia syndrome and bilateral masseter muscle hypertrophy

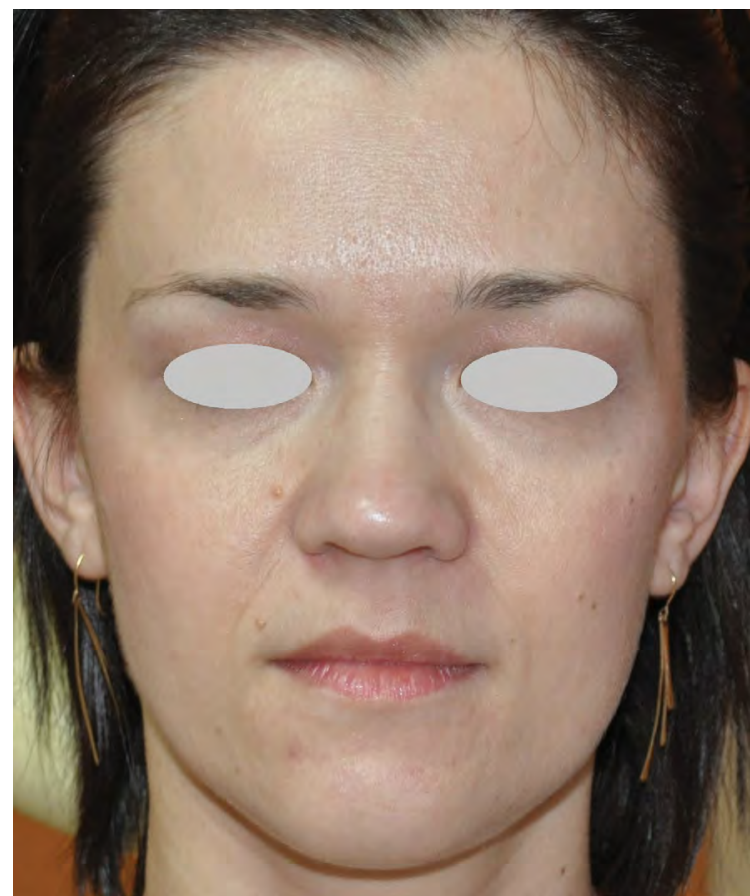

FIGURE 2. Aspect of the patient 3 months after bilateral botulinum toxin injection into the masseter and temporalis muscles. A significant change of the lower face contour is noticeable due to decreased volume of the masseteric regions sensation during the initial month after injection, without any physical signs upon clinical examination. A noticeable loss of volume in the temporal region was encountered in seven patients but was not considered aesthetically displeasing and did not require filling procedures. Twelve patients had a slight asymmetry of the masseteric region that decreased progressively with subsequent injection sessions.

\section{DISCUSSIONS}

In symptomatic bruxism patients, the decrease in pain following neurotoxin injections may be attributed to several effects of the toxin, considering the prevailing factors involved in the etiopathogeneses of the facial arthromyalgia syndrome $(1,4)$. A decrease in the intensity of muscle contraction may play a role, but the known inhibitory effect on the release of inflammation factors may be equally important $(2,5)$. As a result, although most studies report a decrease in the intensity and frequency of bruxism episodes, the complete disappearance of the phenomenon may not be feasible $(4,11,12)$. Nevertheless, a decrease in the associated painful symptoms is achievable using botulinum toxin (12-14).

In our study, the effects on the perceived pain were not conclusive in all patients at one-month post-injection, but a decrease in painful symptoms was obvious in all included patients after three months. This is consistent with the findings of other authors stating that pain was significantly reduced in patients undergoing botulinum toxin injections, with a denoting increase in their life quality $(6,12,13,15)$.

The dose of administered neurotoxin may influence its effectiveness or duration. We used relatively high doses of toxin (100-200 U) injected into both the masseter and temporalis muscles bilaterally, in the areas of muscle hyperactivity. We determined the amount of neurotoxin needed by clinically evaluating the approximate muscle thickness and volume, since larger quantities are necessary for enlarged muscles. Other authors report using similar doses of $100-200 \mathrm{U}$ in total for the masseter and temporalis muscles bilaterally $(13,16)$, or even significantly lower doses of $40 \mathrm{U}$ in total only in the masseter muscles (12), with similar favorable outcomes.

Complications following botulinum toxin injection into the masticatory muscles are not frequently encountered and generally they are mild. Studies report complications like: a decrease in mastication muscle force, insufficient effect, smile asymmetry, xerostomia, bruising, swelling or pain at the injection site, volume loss in the temples area or in the sub-zygomatic region, paradoxical 
bulging of the masseter, headache and allergies $(17,18)$.

Some of the adverse effects are related to the inadequate placement of injections, or administration of inadequate doses $(17,19)$. Palpation of the hyperactivity areas within the muscle ensures placing the injections in the adequate topographic region, while the depth of the injection can be approximated by initially touching the bone and then slightly retracting the needle. In the masseter region, this generally allows avoiding the inadvertent entire administration of neurotoxin into the parotid gland tissue and therefore absence of effect on the masticatory muscles, with possible decreased salivary flow $(18,20)$. Despite an accurate administration, two patients in our study still described a subjective dry mouth sensation, although without any objective signs on clinical examination and without the need for particular treatment, with gradual receding within a month. The possible cause may be the migration of small quantities of the injected neurotoxin due to muscle contraction, but also migration along the pathway of needle withdrawal due to local pressure.

Muscle volume reduction and strength decrease during function are obvious expected outcomes following intramuscular neurotoxin administration. Similar to other studies $(17,18,21)$, the patients in our case series also complained of weakness of the masticatory muscles during the first month, but this did not interfere with performing normal functions.

A decrease in the volume of temporal muscles was another finding in two of the included patients. However, true temporal hollowing was not encountered in any of the patients and therefore, there was no need for filling the temporal region for improving aesthetic appearance. Existing studies also report a very low incidence of this unwanted event (18). Except for the obvious decrease in muscle volume due to the local effects of the botulinum toxin, an additional possible culprit is the inferior movement of the temporal fat pads due to the decreased volume of the masseter muscle lying underneath (18).

Many authors describe the benefits of botulinum toxin for performing a nonsurgical facial contour adaptation $(21,22)$. The decrease in the volume of the masseter muscle was a desired effect in the patients from our case series with obvious accentuated muscle hypertrophy. A small contour change in the outline of the masseteric region was also present in the majority of the patients without initially obvious masseter hypertrophy, but without any negative effects regarding appearance. Asymmetry of the masseteric region following injection was also noticed in more than half of the included patients. The degree of masseteric asymmetry was nevertheless small and gradually improved after subsequent injection sessions.

\section{CONCLUSIONS}

In our experience, botulinum toxin administration proved to be beneficial for controlling the symptomatology associated with bruxism in patients with previous unsuccessful conventional treatment attempts. It represents a simple, effective complementary method of approaching bruxism patients with the purpose of increasing their quality of life.

Conflict of interest: none declared Financial support: none declared

\section{REFERENCES}

1. Laskin DM. The Use of Botulinum Toxin for the Treatment of Myofascial Pain in the Masticatory Muscles. Oral Maxillofac Surg Clin North Am. 2018;30(3):287-289.

2. Connelly ST, Myung J, Gupta R, Tartaglia GM, Gizdulich A, Yang J, Silva R. Clinical outcomes of Botox injections for chronic temporomandibular disorders: Do we understand how Botox works on muscle, pain, and the brain? Int J Oral Maxillofac Surg. 2017;46(3):322-327.

3. Park KS, Lee CH, Lee JW. Use of a botulinum toxin $A$ in dentistry and oral and maxillofacial surgery. J Dent Anesth Pain Med. 2016;16(3):151-157.

4. Beddis H, Pemberton M, Davies S. Sleep bruxism: an overview for clinicians. Br Dent J. 2018;225(6):497-501.
5. Park J, Park HJ. Botulinum Toxin for the Treatment of Neuropathic Pain. Toxins (Basel). 2017;9(9).

6. Villa S, Raoul G, Machuron F, Ferri J, Nicot R. Improvement in quality of life after botulinum toxin injection for temporomandibular disorder. J Stomatol Oral Maxillofac Surg. 2019;120(1):2-6.

7. Oh HM, Chung ME. Botulinum Toxin for Neuropathic Pain: A Review of the Literature. Toxins (Basel). 2015; 7(8):3127-54.

8. Kumar A, Spivakovsky S. Bruxism- is botulinum toxin an effective treatment? Evid Based Dent. 2018;19(2):59.

9. Kwon KH, Shin KS, Yeon SH, Kwon DG. Application of botulinum toxin in maxillofacial field: part I. Bruxism and square jaw. Maxillofac Plast Reconstr Surg. 2019;41(1):38.

10. Long H, Liao Z, Wang Y, Liao L, Lai W. Efficacy of botulinum toxins on bruxism: an evidence-based review. Int Dent J. 2012; 62(1):1-5.

11. De la Torre Canales G, Câmara-Souza MB, do Amaral CF, Garcia RC, Manfredini D. Is there enough evidence to use botulinum toxin injections for bruxism management? A systematic literature review. Clin Oral Investig. 2017;21(3):727-734.

12. Asutay F, Atalay $Y$, Asutay $H, A$ car $A H$. The Evaluation of the Clinical Effects of Botulinum Toxin on Nocturnal Bruxism. Pain Res Manag. 2017;2017:6264146.

13. Denglehem C, Maes JM, Raoul G, Ferri J. Botulinum toxin A: analgesic treatment for 
temporomandibular joint disorders. Rev Stomatol Chir Maxillofac. 2012; 113(1):27-31.

14. Fernández-Núñez T, Amghar-Maach $S$, Gay-Escoda C. Efficacy of botulinum toxin in the treatment of bruxism: Systematic review. Med Oral Patol Oral Cir Bucal. 2019; 24(4):e416-e424.

15. Ghavimi MA, Yazdani J, Afzalimehr A, Ghoreyshizadeh A, Dehnad SV. Effect of injection of botulinum toxin on decreasing the symptoms and signs of masticatory muscles in patients with temporomandibular dysfunction. J Dent Res Dent Clin Dent Prospects. 2019;13(2):128-132.

16. Ondo WG, Simmons JH, Shahid MH, Hashem V, Hunter C, Jankovic J. Onabotulinum toxin-A injections for sleep bruxism: A double-blind, placebo-controlled study. Neurology. 2018;90(7):e559-e564.

17. Peng HP, Peng JH. Complications of botulinum toxin injection for masseter hypertrophy: Incidence rate from 2036 treatments and summary of causes and preventions. J Cosmet Dermatol. 2018; 17(1):33-38.

18. Yeh YT, Peng JH, Peng HP. Literature review of the adverse events associated with botulinum toxin injection for the masseter muscle hypertrophy. $J$ Cosmet Dermatol. 2018;17(5):675-687.

19. Quezada-Gaon N, Wortsman X, Peñaloza $O$, Carrasco JE. Comparison of clinical marking and ultrasound-guided injection of Botulinum type $A$ toxin into the masseter muscles for treating bruxism and its cosmetic effects. J Cosmet Dermatol. 2016; 15(3):238-44.

20. Seo KK. Botulinum Toxin for Asians (Chapter 3, 1st ed.). Singapore: Springer; 2017:107-134.

21. Xie Y, Zhou J, Li H et al. Classification of masseter hypertrophy for tailored botulinum toxin Type A treatment. Plast Reconstr Surg. 2014;134(2):209e-218e.

22. Chang CS, Lin S, Wallace CG, Hsiao YC, Lin CM, Kang GC, Chen ZC, Chen PK, Lo LJ, Chen YR, Chen JP. Masseter Muscle Volume Changes Evaluated by 3-Dimensional Computed Tomography After Repeated Botulinum Toxin A Injections in Patients With Square Facial Morphology. Ann Plast Surg. 2019; 82(1S Suppl 1):S29-S32. 